\title{
2014 CSHP National Awards Program Winners Programme national des prix 2014 de la SCPH : lauréats et lauréates
}

The winner of the Distinguished Service Award (sponsored by Johnson \& Johnson, Family of Companies) is Kelly Babcock (Regina, SK).

The winner of the Isabel E. Stauffer Meritorious Service Award (sponsored by Pharmaceutical Partners of Canada, A Company of the Fresenius Kabi Group) is Lisa Bishop (St John's, NL).
The winners of the New Hospital Pharmacy Practitioner Award (sponsored by Sandoz Canada Inc.) are Lindsay Creamer (London, ON) and Emily Muir (Saint John, NB).

The winner of the Hospital Pharmacy Student Award (cosponsored by the Canadian Society of Hospital Pharmacists [CSHP] and the Canadian Association of Pharmacy Students and Interns [CAPSI] is Erin Chung (Toronto, ON).
CSHP 2015 Hospital Pharmacy Residency Project Award Sponsored by Pharmaceutical Partners of Canada, A Company of the Fresenius Kabi Group

Integration of Smartphones into Clinical Pharmacy Practice: An Evaluation of the Impact on Pharmacists' Efficiency (completed at Vancouver Island Health Authority, British Columbia)

Jessica Power

Management and Leadership Best Practice Award Sponsored by Apotex Inc.

Improving Medication Safety through Implementation of a Best Possible Medication History and Resolved Drug Therapy Problem Tracker Database (completed at Vancouver Island Health Authority, British Columbia)

Sean Spina

Sponsored by Medbuy Corporation

"Simply Indispensable": Transforming Medication Management at the Ross Memorial Hospital (completed at Ross Memorial Hospital, Lindsay, ON)

Susan Fockler

Patient Care Enhancement Award

Sponsored by Pfizer Canada Inc.

Medication-Related Emergency Department Visits in Pediatrics:

A Prospective Observational Study (completed at the IWK Health

Centre, Halifax, NS)

Peter Zed, Neil MacKinnon

Sponsored by Teva Canada Limited

Risk Factors Associated with Venous Thromboembolic Disease in Orthopaedic Surgery Patients: A Retrospective Case Control Study for Quality Analysis (oral presentation) (completed at the Credit Valley Hospital site of Trillium Health Partners, Mississauga, ON) Marie Danette Beechinor, Allan Mills

Pharmacotherapy Best Practices Award

Sponsored by Merck Canada Inc.

Pediatric Assessment of Vancomycin Empiric Dosing (PAVED) (completed at the Children's and Women's Health Centre of British Columbia, Vancouver, BC)

Daniel Rainkie, Mary H H Ensom
Sponsored by Pfizer Canada Inc.

Does Interprofessional Medication Reconciliation from Admission to Discharge Reduce Post-Discharge Patient Emergency Department Visits and Hospital Readmissions? (oral presentation) (completed at the Toronto General Hospital and the TorontoWestern Hospital sites of the University Health Network, Toronto, ON)

Michelle Baker, Derek Leong, Gary Wong, Emily Musing,

Olavo Fernandes

\section{Safe Medication Practices Award} Sponsored by HealthPRO Procurement Services Inc.

Systematic Approach to Evaluate Hazardous Antineoplastic Drugs by a Provincial Healthcare Organization (facilitated poster) (completed at the BC Cancer Agency, British Columbia)

Mário de Lemos, Joan Fabbro

\section{Sponsored by Medbuy Corporation}

Impact of an "Avoid-Heparin" Quality Improvement Program on the Incidence, Clinical Consequences and Resource Use Associated with Heparin-Induced Thrombocytopenia (HIT) (oral presentation) (completed at Sunnybrook Health Sciences Centre, Toronto, ON) Claudia Bucci, Artemis Diamantouros

Specialties in Pharmacy Practice Award Sponsored by Pharmascience Inc. Conversion Process and Cost Savings of a Switch from Intravenous to Subcutaneous Epoetin Alfa in Patients Receiving Hemodialysis (completed at the Manitoba Renal Program, Winnipeg, MB)

Lori Wazny, Amy Sood

Teaching, Learning and Education Award Sponsored by Eli Lilly Canada Inc. 2013 Handbook and Website on Hepatitis C Drug Therapy (completed at the University Health Network, Toronto, ON) Alice Tseng, Pierre Giguère

The award-winning abstracts are published exactly as submitted by the authors and have not undergone any copyediting by the Canadian Journal of Hospital Pharmacy.

Le Journal canadien de la pharmacie hospitalière n'a pas soumis les résumés primés à une révision linguistique et les publie ici tels que remis par les auteurs. 


\section{Integration of Smartphones into Clinical Pharmacy Practice: An Evaluation of the Impact on Pharmacists' Efficiency}

CSHP 2015 Hospital Pharmacy Residency Project Award, sponsored by Pharmaceutical Partners of Canada, A Company of the Fresenius Kabi Group

Jessica M H Power', Sean P Spina ${ }^{1,2}$, David A Forbes ${ }^{3}$, Curtis $K$ Harder ${ }^{1,2,4,5}$, Sherry L Lalli, $i^{1}$ Peter S Loewen ${ }^{2,6}$ ${ }^{\prime}$ Pharmacy Department, Island Health, Victoria, BC

${ }^{2}$ Faculty of Pharmaceutical Sciences, The University of British Columbia, Vancouver, $B C$

${ }^{3}$ Pharmacy Department, Island Health, Nanaimo, BC

${ }^{4}$ Pharmacy Education \& Practice Residency, Island Health, Victoria, BC ${ }^{5}$ Clinical Pharmacy Specialist, Island Health, Victoria, BC

${ }^{6}$ Clinical Pharmacy Specialist, Vancouver Coastal Health, Vancouver, BC

Background: Personal smartphones are used frequently by healthcare practitioners in hospitals to assist in the provision of care. The Vancouver Island Health Authority (VIHA) is one of the first health authorities in Canada to endorse the iPhone ${ }^{\circledR}$ smartphone as a potentially valuable tool for clinical practice. We collaborated with the University of British Columbia (UBC) Faculty of Pharmaceutical Sciences to design and conduct our research. To the best of our knowledge this is the first study of its kind in North America.

Objective: To measure smartphones effect on pharmacists' efficiency, to assess pharmacist acceptance of corporate smartphones, and to investigate how these devices are being utilized.

Methods: This multi-center time-trial, survey, and observational prospective study enrolled 90 pharmacists across eight hospitals on Vancouver Island. Participants performed a time-trial of 22 situational drug information questions before and after receiving an iPhone. They also completed both demographic and satisfaction surveys. A subset of 14 of the 90 pharmacists participated in a pre and post iPhone implementation eight hour direct observation study. Lastly, communication data from the phone service provider was collected and analyzed.

Results: Smartphone use facilitated a statistically significant faster median response time over all the situational time-trial questions combined by approximately six minutes $(2895$ seconds pre-iPhone vs. 2538 seconds post-iPhone) ( $\mathrm{p}=0.039)$. Almost half of pharmacists reported that the smartphone increased their confidence and competence to resolve drug therapy problems. Smartphone use did not significantly influence time spent walking to obtain a resource, use of computers, or in the answering of clinical questions in observation.

Conclusion: Pharmacists readily accepted smartphones into their practice. Despite the lack of impact on direct observation measures of workflow, smartphones improved pharmacists' efficiency in the answering of hypothetical questions. This research provides sufficient evidence to continue to support the use of smartphones within VIHA's pharmacy department.

Key words: phone, pharmacist, computers (handheld), clinical pharmacy information systems, communication, technology

\section{Improving Medication Safety through Implementation of a Best Possible Medication History and Resolved Drug Therapy Problem Tracker Database}

Management and Leadership Best Practice Award, sponsored by Apotex Inc.

Sean P Spina $a^{1,2}$, Glennys La Rose ${ }^{3}$

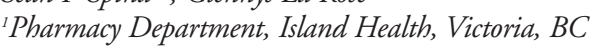

${ }^{2}$ Faculty of Pharmaceutical Sciences, The University of British Columbia, Vancouver, $B C$

${ }^{3}$ Pharmacy Department, Island Health, Nanaimo, BC

Background: A need was identified to improve the process of documenting patient's best possible medication history (BPMH) on admission, transfer between units/facilities and on discharge and to track the number of drug therapy problems resolved (rDTP). Initially the Microsoft Access database was developed as part of a research project between the University of Victoria and the Vancouver Island Health Authority. Once the research project was completed, the value of the database was realized by senior leadership and the database was expanded to meet the needs of the pharmacists and health authority.

Objectives: To develop a Microsoft Access database to capture clinical pharmacy Key Performance Indicators (cpKPIs) and electronically document patient's admission BPMH, transfer BPMTL and discharge BPMDL.

Methods: Traditionally pharmacists handwrote patient's BPMHs and physicians would cross out medications with a felt marker and hand write additional medication orders as required. A Microsoft Access database was developed allowing pharmacists to electronically document patient's admission BPMH, transfer best possible medication transfer list (BPMTL) and discharge best possible medication discharge list (BPMDL), standardizing the forms used by pharmacists and physicians in combination with documenting resolved drug therapy problems.

Results: Since the database has been included into clinical pharmacists daily practice the number of patient's BPMH completed has increased along with the number of $\mathrm{rDTPs}$ resolved.

Conclusion: The VIHA Pharmacy Department identified a care gap in 2010 which resulted in the development of a Microsoft Access Database allowing for the electronic documentation of BPMHs and rDTPs. This data has been instrumental in advocating for essential role that clinical pharmacists play on the care team.

Key words: Medication Reconciliation, Best Possible Medication History, Resolved Drug Therapy Problems, Pharmacist, Technology

\section{"Simply Indispensable": Transforming Medication Management at the Ross Memorial Hospital \\ Management and Leadership Best Practice Award, sponsored by Medbuy Corporation \\ Susan Fockler \\ Ross Memorial Hospital, Lindsay, ON}

The medication management system at the Ross Memorial Hospital has been completely transformed since the CSHP 2015 goals were introduced in 2007. Improvements in medication use and safety have been achieved through initiatives in drug distribution, administration and clinical services. In the last seven years the department has gone from a traditional drug distribution system run with a stand-alone computer system to a full unit dose and IV additive system run by pharmacy technicians. Both Pharmacist and Pharmacy Technicians have decentralized to enhance collaboration with other healthcare professionals and to provide direct patient care.

In 2009 our Pharmacy Department developed our mission and vision to clearly define and communicate our intended role at the Ross Memorial Hospital. We wanted to emphasize that Pharmacy's purpose is to collaborate with other healthcare professionals to provide quality patient care, to develop systems that enhance safety in all steps of the medication process and to contribute our unique skills and knowledge as pharmacists and pharmacy technicians. At that time we met less than $10 \%$ of the CSHP 2015 goals. This award application outlines how our 
2014 CSHP NATIONAL AWARDS PROGRAM / PROGRAMME NATIONAL DES PRIX 2014 DE LA SCPH

Pharmacy Department has partially or fully achieved $77 \%$ of these safety objectives since their introduction. These achievements have been achieved through visionary leadership, working collaboratively with key stakeholders and turning challenges into opportunities.

\author{
Medication-Related Emergency Department Visits \\ in Pediatrics: A Prospective Observational Study \\ Patient Care Enhancement Award, sponsored by \\ Pfizer Canada Inc. \\ Peter J Zed ${ }^{l, 2}$, Karen JL Black, ${ }^{3,4}$ Eleanor A Fitzpatrick, \\ Stacy Ackroyd-Stolarz ${ }^{3,5}$, Nancy G Murphy ${ }^{3,5,6}$, Janet A Curran ${ }^{3,4,7}$, \\ Neil J MacKinnon ${ }^{8}$, Doug Sinclair ${ }^{9}$ \\ ${ }^{1}$ Faculty of Pharmaceutical Sciences, University of British Columbia, \\ Vancouver, $B C$ \\ ${ }^{2}$ Department of Emergency Medicine, Faculty of Medicine, University of \\ British Columbia, Vancouver, $B C$ \\ ${ }^{3}$ Department of Emergency Medicine, Dalhousie University, Halifax, NS \\ ${ }^{4}$ IWK Health Centre, Halifax, NS \\ ${ }^{5}$ Capital Health, Halifax, NS \\ ${ }^{6}$ IWK Regional Poison Control Centre, Halifax, NS \\ ${ }^{7}$ School of Nursing, Dalhousie University Halifax, NS \\ ${ }^{8}$ James L Winkle College of Pharmacy, University of Cincinnati, \\ Cincinnati, $\mathrm{OH}$ \\ 'St Michael's Hospital, Toronto, ON
}

Background: There are few data on the rate and characterization of medication-related visits (MRV) to the emergency department (ED) in pediatric patients and none available in Canada. We sought to evaluate the frequency, severity, preventability and classification of MRVs to the $\mathrm{ED}$ in pediatrics in a large tertiary-care teaching hospital.

Methods: We performed a prospective observational study of pediatric patients presenting to the ED over a 1-year period. A medicationrelated ED visit was identified using pharmacist assessment, emergency physician assessment and an independent adjudication committee.

Results: 2028 patients were enrolled (mean age $6.1 \pm 5.0$ years, 52.6\% female). A MRV was identified in 163 patients, $8.0 \% \quad(95 \% \mathrm{CI}$ 7.0-9.3\%) of which $65.0 \%$ (95\%CI 57.2-72.3\%) were deemed preventable. Severity was classified as mild, moderate and severe in $8.6 \%$ (95\%CI 4.8-14.0\%), 85.9\% (95\%CI 79.6-90.8\%), and 5.5\% (95\%CI 2.6-10.2\%) of cases, respectively. The most common reasons for MRVs were adverse drug reactions 26.4\% (95\%CI 19.8-33.2\%), subtherapeutic dose $19.0 \%$ (95\%CI 13.3-25.9\%) and non-adherence $17.2 \%$ (95\%CI $11.7-23.9 \%)$. The probability of admission was significantly higher among patients with a MRV (OR 6.5, 95\%CI 4.3-9.6) and if admitted the median (IQR) length of stay was longer (3.0 (5.0) days vs. 1.5 (2.5) days, $\mathrm{p}=0.02$ ).

Conclusion: A medication-related cause was found in approximately one of every twelve pediatrics ED visits, of which two-thirds were deemed preventable. Pediatric patients that present to the ED with a MRV are more likely to be admitted to hospital and when admitted have a longer LOS.

\author{
Risk Factors Associated with Venous \\ Thromboembolic Disease in Orthopaedic Surgery \\ Patients: A Retrospective Case Control Study \\ for Quality Analysis (oral presentation) \\ Patient Care Enhancement Award, sponsored by \\ Teva Canada Limited \\ Manal Rostom ${ }^{I}$, Marie Danette Beechinor ${ }^{2}$, Allan Mills',3, \\ Blair Ernst
}

'University of Waterloo School of Pharmacy, Kitchener, ON ${ }^{2}$ Credit Valley Hospital and Trillium Health Centre, Mississauga, $O N$ ${ }^{3}$ University of Toronto, Toronto, $\mathrm{ON}$

Rationale: The rate of symptomatic venous thromboembolism (VTE) at our institution in the 2011 and 2012 calendar years was $1.75 \%$ following total knee arthroplasty (TKA), 1.73\% following total hip arthroplasty (THA), and $2.07 \%$ following hip fracture surgery (HFS), higher than reported rates despite appropriate thromboprophylaxis, which are $0.80 \%$ and $0.35 \%$, respectively, in the first 14 days. This was an opportunity to systematically explore all modifiable and process related risk factors for VTE to determine if there were any potential quality related improvements.

Objectives: To determine the impact of modifiable perioperative clinical practices on the development of VTE and to demonstrate the importance of systematic analysis of outcome data for quality improvement.

Methods: This retrospective case control study included patients 18 years of age or older, undergoing a TKA, THA, or HFS at our institution in 2011 and 2012. For each case, 3 controls without VTE were matched for sex, age ( \pm 5 years), surgery, and year of surgery. Data analysis was conducted using stepwise multiple regression analysis.

Results: VTE was significantly and independently predicted by the time to initiation of VTE prophylaxis postoperatively $(\mathrm{R}=0.240$, $\mathrm{p}=0.010$ ). The $\mathrm{R} 2$ value for this model was 0.057 , showing $5.7 \%$ of the variability in diagnosis of VTE was due to this variable. The $\mathrm{R}$ value shows a weak but significant correlation between time to the first dose of prophylaxis and the diagnosis of VTE. Patients diagnosed with symptomatic VTE received the first dose of prophylaxis an average of 24.6 (SD 11.1) hours post-operatively, and controls 18.6 (SD 10.4) hours post-operatively.

Conclusion: The time to the first dose of prophylaxis is an important and modifiable predictor for symptomatic VTE post orthopedic surgery. Time to first dose should be considered when developing systems to improve patient care.

\section{Pediatric Assessment of Vancomycin Empiric Dosing (PAVED) \\ Pharmacotherapy Best Practices Award, sponsored by Merck Canada Inc.}

Daniel Rainkie ${ }^{l}$, Mary H H Ensom ${ }^{l, 2}$

'Pharmacy Department, Children's and Women's Health Centre

of British Columbia, Vancouver, BC

${ }^{2}$ Faculty of Pharmaceutical Sciences, University of British Columbia, Vancouver, $B C$

Background: Pediatric studies and anecdotal experience suggest that current empiric vancomycin dosing does not reach serum trough concentration targets of at least $10 \mathrm{mg} / \mathrm{L}$ for uncomplicated infections or $15-20 \mathrm{mg} / \mathrm{L}$ for serious or complicated infections.

Methods: Data were extracted from medical records of 200 patients aged 1 month to 18 years, who received intravenous vancomycin and had at least 2 evaluable serum concentrations.

Results: Trough vancomycin concentrations of $10-15 \mathrm{mg} / \mathrm{L}$ and $15-20$ $\mathrm{mg} / \mathrm{L}$ were achieved in $25(29 \%)$ and $2(2 \%)$ of patients receiving 15 $\mathrm{mg} / \mathrm{kg}$ IV q6h and $22(20 \%)$ and $9(8 \%)$ of patients receiving 20 $\mathrm{mg} / \mathrm{kg}$ IV q $8 \mathrm{~h}$, respectively. Patients were stratified into four age groups (1 month-1 year, 1-6 years, 6-13 years and 13-18 years). Median (IQR) pharmacokinetic parameters were: elimination rate constant 0.25 (0.09), 0.29 (0.07), $0.24(0.10)$ and $0.22(0.07) \mathrm{h}-1$; volume of distribution $0.55(0.20), 0.60(0.23), 0.45(0.23)$ and $0.45(0.18) \mathrm{L} / \mathrm{kg}$; and half-life $2.8(1.1), 2.37(0.5), 2.9(1.1)$ and $3.2(1.0) \mathrm{h}$, respective- 
ly. Median (IQR) AUCs were 465 (178), 338 (132), 501 (197) and 519 (211) and population-estimated AUCs were 62 (41), 83 (56), 235 (81) and 316 (88) $\mathrm{mg}^{*} \mathrm{~h} / \mathrm{L}$ ( $\mathrm{p}<0.05$ for all groups).

Conclusions: We therefore recommend the following to undergo further testing as initial vancomycin dosing regimens: $70 \mathrm{mg} / \mathrm{kg} /$ day and $90 \mathrm{mg} / \mathrm{kg} /$ day divided $\mathrm{q} 6 \mathrm{~h}$ for troughs of $10-15 \mathrm{mg} / \mathrm{L}$ and $15-20 \mathrm{mg} / \mathrm{L}$, respectively (patients 1 month- 6 years) and $60 \mathrm{mg} / \mathrm{kg} /$ day divided $\mathrm{q} 8 \mathrm{~h}$ and $70 \mathrm{mg} / \mathrm{kg} /$ day divided $\mathrm{q} 6 \mathrm{~h}$, respectively (patients $>6$ years). Furthermore, population estimates grossly underestimate vancomycin AUC in patients 1 month-18 years old and thus patientspecific parameters are required.

\section{Does Interprofessional Medication Reconciliation from Admission to Discharge Reduce Post- Discharge Patient Emergency Department Visits and Hospital Readmissions? (oral presentation) Pharmacotherapy Best Practices Award, sponsored by Pfizer Canada Inc}

Michelle Baker', Chaim M Bell, ${ }^{3,4,}$, Wei Xiong $g^{l}$,Edward Etchells ${ }^{4,5}$, Peter Rossos ${ }^{l, 4}$, Kaveh Shojania ${ }^{5}$, Kelly Lanel, Tim Tripp ${ }^{l}$, Mary Lam ${ }^{l}$, Kimindra Tiwana ${ }^{6}$, Nita Dhir', Derek Leongl, Gary Wong $g^{1,4}$, Jin Huh ${ }^{1,4}$, Emily Musing ${ }^{1,4}$, Olavo Fernandes ${ }^{l, 4}$

${ }^{I}$ University Health Network, Toronto, ON

${ }^{2}$ Mount Sinai Hospital, Toronto, ON

${ }^{3}$ St Michael's Hospital, Toronto, ON

${ }^{4}$ University of Toronto, Toronto, $O N$

${ }^{5}$ Sunnybrook Health Sciences Centre, Toronto, ON

${ }^{6}$ Institute for Safe Medication Practices Canada, Toronto, ON

Rationale: Medication reconciliation has been shown to reduce potential adverse drug events, but its specific impact on post-discharge hospital readmission is still not known.

Objective: To evaluate the impact of integrated interprofessional (pharmacist-prescriber) medication reconciliation on patient emergency department visits and hospital readmissions.

Study Design and Methods: In this observational cohort study at a tertiary-care hospital, patients discharged by General Internal Medicine (GIM) were identified through administrative databases. The intervention group (patients receiving interprofessional admission to discharge reconciliation supported by an electronic platform) was compared to a control group. The outcome was defined as a composite of emergency department or hospital readmissions within 30 days of the index discharge. A multivariate logistic regression model was used to adjust for age, gender, number of medications, and LACE index.

Results: From 2007-2011, a total of 9,931 unique GIM patient visits $(\mathrm{n}=8678$ patients) met the criteria of the study. The main analysis did not detect a difference in outcomes between the intervention group (540/2541) and control (1423/7390) for the primary endpoint. The adjusted odd's ratio was 1.058 (21.25\% vs. $19.26 \%$, 95\% CI 0.945 $1.19, \mathrm{p}=0.326$ ). After propensity score adjustment, the relative risk of readmission was 0.88 (16.7\% intervention vs. $18.9 \%$ control, $95 \%$ CI $0.59-1.32, \mathrm{p}=0.535)$. Increasing number of medications, LACE index score, as well as male gender were independently correlated with a higher risk of hospital visits. Also, subgroup analyses of high-risk groups: patients $\geq 65$ years, LACE index $\geq 10$, those on high-alert medications, and $\geq 10$ medications did not detect a statistically significant outcome difference between groups.

Conclusion: A 5 year observational evaluation of interprofessional medication reconciliation did not detect a difference in 30 day postdischarge patient hospital visits. Future prospective studies could focus on an enhanced reconciliation intervention bundle on avoidable "medication-related" hospital admissions and post-discharge adverse drug events.

\section{Systematic Approach to Evaluate Hazardous Antineoplastic Drugs by a Provincial Healthcare Organization (facilitated poster) \\ Safe Medication Practices Award, sponsored by HealthPRO Procurement Services Inc.}

Mário de Lemos', Nadine Badry², Joan Fabbro ${ }^{3}$

${ }^{\prime} B C$ Cancer Agency, Vancouver, BC

${ }^{2} B C$ Cancer Agency, Victoria, $B C$

${ }^{3} B C$ Cancer Agency, Kelowna, BC

Rationale: The US National Institute for Occupational Safety and Health (NIOSH) list of hazardous drugs and evaluation criteria provide a foundation to help identify hazardous formulary drugs. However, we needed to develop further guiding principles to adopt the NIOSH guidelines.

Objective: To identify hazardous oncology drugs used by the $\mathrm{BC}$ Cancer Agency (BCCA).

Study Design/Methods: Three guiding principles were developed.

Inherent toxicity vs. occupational exposure NIOSH defines hazard based on inherent drug toxicity. Safe handling policies are driven by workers' protection from this toxicity before considering the resource-dependent operations to minimize occupational exposure. We found no strong evidence to support differentiating hazard levels (high, low).

NIOSH reviews We assumed drugs marketed pre-2004 were reviewed since NIOSH List 2004 was compiled from major US organizations. Drugs marketed post-2004 were ascertained with NIOSH lists (2010, 2012) and proposed lists (2004-12).

Drug evaluation NIOSH decisions are based on its evaluation criteria and external consultations. Based on the NIOSH proposed changes (2006, 2011), fulfilling the criteria would not necessarily make a drug hazardous.

Results: We identified hazardous drugs by assessing their inherent toxicity and developed safe handling policies based on occupational exposure related to dosage form. Our list consists of drugs in the current NIOSH list and assessed by BCCA because NIOSH review was not confirmed. A drug is hazardous if designated by NIOSH. Other drugs are hazardous if they fulfill the NIOSH criteria or used primarily as antineoplastic but insufficient information to evaluate with NIOSH criteria.

Conclusion: BCCA has adopted an approach within the NIOSH framework and it follows the precautionary principle when there is risk of harm affecting individuals not directly benefiting from these hazardous drugs.

\section{Impact of an "Avoid-Heparin" Quality Improvement Program on the Incidence, Clinical Consequences and Resource Use Associated with Heparin-Induced Thrombocytopenia (HIT) \\ Safe Medication Practices Award, sponsored by Medbuy Corporation}

Kelly McGowann, Joy Makari', ${ }^{1,3}$, Peter Rempel', Claudia Bucci, ${ }^{1,2,3}$, Artemis Diamantouros $^{1,2,3}$, William Geerts ${ }^{1,2}$

${ }^{1}$ Faculty of Medicine, University of Toronto, Toronto, $O N$

${ }^{2}$ Sunnybrook Health Sciences Centre, Toronto, ON

${ }^{3}$ Leslie Dan Faculty of Pharmacy, University of Toronto, Toronto, ON 
Rationale: Unfractionated heparin (UFH) is one of the most common drugs used in hospitals but produces heparin-induced thrombocytopenia (HIT) in up to $5 \%$ of exposed patients. HIT leads to thrombosis, amputations, death, and massive use of resources. Can HIT be prevented?

Objective: To examine the impact of a formal hospital-wide strategy to limit exposure to UFH on the incidence of HIT and its consequences.

Study Design and Methods: A multi-disciplinary task force reviewed the literature for each type of heparin exposure and prepared recommendations for practice modifications. A comprehensive "Avoid-Heparin Program" was implemented at a Canadian teaching hospital in 2006 as a specific attempt to reduce HIT and improve patient safety. This involved replacing intravenous(IV) and subcutaneous (S/C) UFH with LMWH for prophylactic and therapeutic indications and removing UFH from arterial and central venous lines. The program was evaluated by retrospective chart review.

Measures: All cases of suspected HIT 2003-2011 were adjudicated using explicit criteria into Negative, HIT and HIT with thrombosis (HITT). Outcomes in the Pre-Intervention Phase (2003-05) were compared to those in the Avoid-Heparin Phase (2007-11).

Results: The annual number of suspected HIT cases decreased 34\% from the Pre-Intervention Phase to the Avoid-Heparin Phase $(p<0.001)$. Adjudicated HIT decreased $73 \%$ from 11 to $3 / 10,000$ admissions $(p<0.001)$ and HITT decreased $80 \%$ from 5 to $1 / 10,000$ admissions $(p<0.001)$. The hospital expenditure on HIT-safe anticoagulants also decreased $41 \%$ in the Avoid-Heparin Phase; this led to mean cost saving of $\$ 257,910$ /year.

Conclusion: To our knowledge, this is the first quality improvement study demonstrating the success of a hospital-wide HIT prevention strategy. Implementation of a simple heparin avoidance intervention can lead to a dramatic decrease in the burden of HIT, the costs of HIT care, and ultimately to improved patient outcomes.

\section{Conversion Process and Cost Savings of a Switch from Intravenous to Subcutaneous Epoetin Alfa in Patients Receiving Hemodialysis \\ Specialties in Pharmacy Practice Award, sponsored by Pharmascience Inc.}

Lori Wazny, Amy Sood, Mauro Verrelli

Manitoba Renal Program and Manitoba e-Health Services, Winnipeg, MB

Purpose: The purpose of this study was to describe our conversion process from intravenous (IV) to subcutaneous (SC) epoetin alfa and to evaluate drug costs pre and post switch.

Description of the Project: This retrospective analysis compared epoetin alfa drug costs during a six-month period of IV usage (July-Dec. 2010, Period 1) to a six-month period of SC usage (July-Dec. 2011, Period 2) in all patients receiving in-center hemodialysis (HD) in Winnipeg, Manitoba, Canada. Data were collected from quarterly counts of HD patients receiving epoetin alfa and monthly inventory billing records.
Project Results: 622 HD patients who received IV epoetin alfa (Period 1) were compared to 609 HD patients who received SC epoetin alfa (Period 2). Over $98 \%$ of patients were successfully converted to SC epoetin alfa administration. The average weekly cost of epoetin alfa was $\$ 173.02 \mathrm{CDN}$ per patient during the IV period versus $\$ 151.20$ $\mathrm{CDN}$ per patient during the SC period. This equated to a yearly cost savings of $\$ 1,135 \mathrm{CDN}$ per patient with SC epoetin alfa.

Conclusion: The switch from epoetin alfa IV to epoetin alfa SC was successfully implemented in a large hemodialysis program and realized significant cost savings

\section{Handbook and Website on Hepatitis C Drug Therapy \\ Teaching, Learning and Education Award, sponsored by Eli Lilly Canada Inc.}

Alice Tseng ${ }^{1,2}$, Pierre Giguère, ${ }^{3,4}$, Sanjeev Sockalingam ${ }^{5,6}$

${ }^{1}$ Immunodeficiency Clinic, University Health Network, Toronto, ON

${ }^{2}$ Leslie Dan Faculty of Pharmacy, University of Toronto, Toronto, ON

Immunodeficiency Clinic, The Ottawa Hospital, Ottawa, ON

${ }^{4}$ The Ottawa Hospital Research Institute, Ottawa, ON

${ }^{5}$ Program in Medical Psychiatry, University Health Network, Toronto, ON ${ }^{6}$ Departments of Psychiatry and Medicine, University of Toronto, Toronto, $O N$

Purpose: With the recent advent of directly acting antiviral (DAA) agents, the treatment of hepatitis $\mathrm{C}(\mathrm{HCV})$ has been revolutionized. Cure rates for $\mathrm{HCV}$ genotype 1 with triple therapy consisting of a DAA plus pegylated-interferon and ribavirin are significantly higher than prior success rates with dual treatment. New agents are being developed at a rapid pace, with at least 3 new DAAs expected to come to market in the next year, thus adding to the complexity of treatment. Practitioners caring for hepatitis C-infected patients now need to consider drug interactions initiating triple therapy for hepatitis $\mathrm{C}$, particularly for patients with comorbid illnesses including HIV infection. A hepatitis $\mathrm{C}$ handbook and website for health care professionals were created to address this need.

Methods: Information was retrieved via a MEDLINE search for English language human studies and case reports for the years 2011present. MeSH headings included: "hepatitis C", "boceprevir", "telaprevir" and "drug interactions". Additional information was obtained from abstracts of international and national hepatitis conferences, review articles, and drug manufacturers.

Results: An 88-page pocket handbook and website were developed and launched in January 2013. The handbook contains information on pharmacologic and pharmacokinetic properties of licensed DAAs as well as several drug interaction tables with commonly prescribed classes of medication. The website is updated regularly as new data emerge, and already includes several additional drug interaction tables including interactions with investigational HCV agents as well as a downloadable e-book version of the HCV handbook.

Conclusions: A handbook and website on HCV drug therapy were successfully developed and disseminated to health care practitioners nationally and internationally. 